\title{
Commentary on "On Intuitionistic Fuzzy Copula Aggregation Operators in Multiple-Attribute Decision Making"
}

\author{
Arshdeep Kaur $^{1}$ • Amit Kumar ${ }^{1}$
}

Received: 12 September 2018 / Accepted: 10 June 2020 / Published online: 17 June 2020

(C) Springer Science+Business Media, LLC, part of Springer Nature 2020

\section{Introduction}

Tao et al. [1, Definition 4, p. 613] proposed the operational law (1) and the operational law (2) respectively to evaluate the sum and the multiplication of two IFVs $\alpha_{1}=\left\langle\mu_{\alpha_{1}}, \nu_{\alpha_{1}}\right\rangle$ and $\alpha_{2}=\left\langle\mu_{\alpha_{2}}, \nu_{\alpha_{2}}\right\rangle$.

$\alpha_{1} \oplus_{c} \alpha_{2}=\left\langle 1-\phi^{-1}\left[\phi\left(1-\mu_{\alpha_{1}}\right)+\phi\left(1-\mu_{\alpha_{2}}\right)\right], \phi^{-1}\left[\phi\left(\nu_{\alpha_{1}}\right)+\phi\left(\nu_{\alpha_{2}}\right)\right]\right\rangle$

$\alpha_{1} \otimes_{c} \alpha_{2}=\left\langle\phi^{-1}\left[\phi\left(\mu_{\alpha_{1}}\right)+\phi\left(\mu_{\alpha_{2}}\right)\right], 1-\phi^{-1}\left[\phi\left(1-\nu_{\alpha_{1}}\right)+\phi\left(1-\nu_{\alpha_{2}}\right)\right]\right\rangle$

Hence, Tao et al. [1] proposed the operational law (3) to evaluate the sum of " $n$ " IFVs $\alpha_{i}=\left\langle\mu_{\alpha_{i}}, \nu_{\alpha_{i}}\right\rangle, i=1,2, \ldots, n$ and the operational law (4) to evaluate the multiplication of $n$ IFVs $\alpha_{i}=\left\langle\mu_{\alpha_{i}}, \nu_{\alpha_{i}}\right\rangle, i=1,2, \ldots, n$.

$$
\begin{aligned}
& \oplus_{c_{i=1}^{n}}^{n} \alpha_{i}=\left\langle 1-\phi^{-1}\left(\sum_{i=1}^{n} \phi\left(1-\mu_{\alpha_{i}}\right)\right) \phi^{-1}\left(\sum_{i=1}^{n} \phi\left(\nu_{\alpha_{i}}\right)\right)\right\rangle \\
& \otimes_{c_{i=1}}^{n} \alpha_{i}=\left\langle\phi^{-1}\left(\sum_{i=1}^{n} \phi\left(\mu_{\alpha_{i}}\right)\right), 1-\phi^{-1}\left(\sum_{i=1}^{n} \phi\left(1-\nu_{\alpha_{i}}\right)\right)\right\rangle
\end{aligned}
$$

Also, using the operational law (3) and the operational law (4), Tao et al. [1, Theorem 7, p. 616] proposed the IFCAAO (5) to aggregate $n$ IFVs $\alpha_{i}=\left\langle\mu_{\alpha_{i}}, \nu_{\alpha_{i}}\right\rangle, i=1,2, \ldots, n$ by considering " $w_{i}$ " as the normalized weight associated with the intuitionistic fuzzy value (IFV) " $\alpha_{i}=\left\langle\mu_{\alpha_{i}}, \nu_{\alpha_{i}}\right\rangle$."

$$
\begin{aligned}
& \bigoplus_{c_{i=1}}^{n}\left(w_{i} \otimes_{c} \alpha_{i}\right) \\
& \quad=\left\langle 1-\phi^{-1}\left(\sum_{i=1}^{n} w_{i} \times \phi\left(1-\mu_{\alpha_{i}}\right)\right), \phi^{-1}\left(\sum_{i=1}^{n} w_{i} \times \phi\left(\nu_{\alpha_{i}}\right)\right)\right\rangle
\end{aligned}
$$

where $\phi$ is a strictly decreasing function such that $\phi(1)=0$, $\phi(0)=\infty, \phi^{-1}(0)=1$, and $\phi^{-1}(\infty)=0[1$, Proof of Theorem 2, p. 613].

Arshdeep Kaur

arshdeep.math@gmail.com

School of Mathematics, Thapar Institute of Engineering \& Technology (Deemed to be University), Patiala, Punjab, India
The aim of this commentary is to make the researchers aware that

(i) The operational law (3), proposed by Tao et al. [1, Definition 4, p. 613] to evaluate the sum of $n$ IFVs $\alpha_{i}=\left\langle\mu_{\alpha_{i}}, \nu_{\alpha_{i}}\right\rangle, i=1,2, \ldots, n$, can be used only if $\alpha_{i} \neq$ $\langle 1,0\rangle$ for any $i$.

(ii) The operational law (4), proposed by Tao et al. [1, Definition 4, p. 613] to evaluate the multiplication $n$ IFVs $\alpha_{i}=\left\langle\mu_{\alpha_{i}}, \nu_{\alpha_{i}}\right\rangle, i=1,2, \ldots, n$, can be used only if $\alpha_{i} \neq\langle 0,1\rangle$ for any $i$.

(iii) The IFCAAO (5), proposed by Tao et al. [1, Theorem 7 , p. 616] to aggregate $n$ IFVs $\alpha_{i}=\left\langle\mu_{\alpha_{i}}, \nu_{\alpha_{i}}\right\rangle, i=1,2, \ldots$, $n$, can be used only if $\alpha_{i} \neq\langle 1,0\rangle$ for any $i$.

\section{Limitation of Tao et al.'s Operational Laws}

In this section, it is shown that

(i) The operational law (3), proposed by Tao et al. [1] to evaluate the sum of $n$ IFVs $\alpha_{i}=\left\langle\mu_{\alpha_{i}}, \nu_{\alpha_{i}}\right\rangle, i=1,2, \ldots$, $n$, can be used only if $\alpha_{i} \neq\langle 1,0\rangle$ for any $i$.

(ii) The operational law (4), proposed by Tao et al. [1] to evaluate the multiplication of $n$ IFVs $\alpha_{i}=\left\langle\mu_{\alpha_{i}}, \nu_{\alpha_{i}}\right\rangle$, $i=1,2, \ldots, n$, can be used only if $\alpha_{i} \neq\langle 0,1\rangle$ for any $i$.

\section{Limitation of Tao et al.'s Operational Law to Evaluate the Sum of Finite Number of IFVs}

In this section, it is shown that if one of the $n$ IFVs $\alpha_{i}=\left\langle\mu_{\alpha_{i}}, \nu_{\alpha_{i}}\right\rangle, i=1,2, \ldots, n$ will be $\langle 1,0\rangle$. Then, the sum of all the $n$ IFVs will also be $\langle 1,0\rangle$ i.e., if $\alpha_{p}=\left\langle\mu_{\alpha_{p}}, \nu_{\alpha_{p}}\right\rangle$ $=\langle 1,0\rangle$ then the sum of the $n$ IFVs $\alpha_{i}=\left\langle\mu_{\alpha_{i}}, \nu_{\alpha_{i}}\right\rangle, i=1,2$, $\ldots, n$ will be independent from the remaining " $(n-1)$ " IFV 
values $\alpha_{i}=\left\langle\mu_{\alpha_{i}}, \nu_{\alpha_{i}}\right\rangle, i=1,2, \ldots, p-1, p+1, \ldots n$. Hence, the operational law (3), proposed by Tao et al. [1] to evaluate the sum of $n$ IFVs $\alpha_{i}=\left\langle\mu_{\alpha_{i}}, \nu_{\alpha_{i}}\right\rangle, i=1,2, \ldots, n$, can be used only if $\alpha_{i} \neq\langle 1,0\rangle$ for any $i$.

The operational law (6) represents an alternative form of the operational law (3).

$$
\oplus_{c_{i=1}^{n} \alpha_{i}}^{n}=\left\langle 1-\phi^{-1}\left(\phi\left(1-\mu_{\alpha_{p}}\right)+\sum_{\substack{i=1 \\ i \neq p}}^{n} \phi\left(1-\mu_{\alpha_{i}}\right)\right), \phi^{-1}\left(\phi\left(\nu_{\alpha_{p}}\right)+\sum_{\substack{i=1 \\ i \neq p}}^{n} \phi\left(\nu_{\alpha_{i}}\right)\right)\right\rangle
$$

Let $\alpha_{p}=\left\langle\mu_{\alpha_{p}}, \nu_{\alpha_{p}}\right\rangle=\langle 1,0\rangle$, i.e., $\mu_{\alpha_{p}}=1$ and $\nu_{\alpha_{p}}=0$. Then, using the operational law (6),

$$
\begin{aligned}
& \oplus_{c i=1}^{n} \alpha_{i}=\left\langle 1-\phi^{-1}\left(\phi(1-1)+\sum_{\substack{i=1 \\
i \neq p}}^{n} \phi\left(1-\mu_{\alpha_{i}}\right)\right), \phi^{-1}\left(\phi(0)+\sum_{\substack{i=1 \\
i \neq p}}^{n} \phi\left(\nu_{\alpha_{i}}\right)\right)\right\rangle \\
& =\left\langle 1-\phi^{-1}\left(\phi(0)+\sum_{\substack{i \neq 1 \\
i \neq p}}^{n} \phi\left(1-\mu_{\alpha_{i}}\right)\right), \phi^{-1}\left(\phi(0)+\sum_{\substack{i \neq 1 \\
i \neq p}}^{n} \phi\left(\nu_{\alpha_{i}}\right)\right)\right\rangle
\end{aligned}
$$

Using the existing relation $\phi(0)=\infty[1$, Proof of Theorem 2, p. 613],

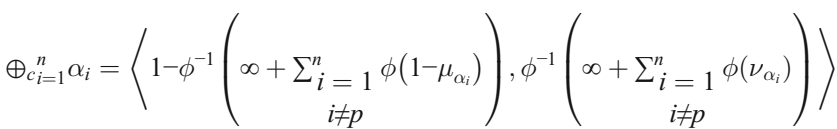
$=\left\langle 1-\phi^{-1}(\infty), \phi^{-1}(\infty)\right\rangle$

Using the existing relation $\phi^{-1}(\infty)=0[1$, Proof of Theorem 2, p. 613],

$\oplus_{c_{i=1}^{n}}^{n} \alpha_{i}=\langle 1-0,0\rangle$

$=\langle 1,0\rangle$.

\section{Limitation of Tao et al.'s Operational Law to Evaluate the Multiplication of IFVs}

In this section, it is shown that if one of the $n$ IFVs $\alpha_{i}=\left\langle\mu_{\alpha_{i}}, \nu_{\alpha_{i}}\right\rangle, i=1,2, \ldots, n$ will be $\langle 0,1\rangle$, then the multiplication of all the $n$ IFVs $\alpha_{i}=\left\langle\mu_{\alpha_{i}}, \nu_{\alpha_{i}}\right\rangle, i=1,2, \ldots, n$ will also be $\langle 0,1\rangle$, i.e., if $\alpha_{p}=\langle 0,1\rangle$, then the multiplication of the $n$ IFVs $\alpha_{i}=\left\langle\mu_{\alpha_{i}}, \nu_{\alpha_{i}}\right\rangle, i=1,2, \ldots, n$ will be independent from the remaining $(n-1)$ IFV values $\alpha_{i}=\left\langle\mu_{\alpha_{i}}, \nu_{\alpha_{i}}\right\rangle, i=1,2, \ldots$, $p-1, p+1, \ldots, n$. Hence, the operational law (4), proposed by Tao et al. [1] to evaluate the multiplication of $n$ IFVs $\alpha_{i}=\left\langle\mu_{\alpha_{i}}, \nu_{\alpha_{i}}\right\rangle, i=1,2, \ldots, n$, can be used only if $\alpha_{i} \neq\langle 0,1\rangle$ for any $i$.

The operational law (7) represents an alternative form of the operational law (4). $\otimes_{c i=1}^{n} \alpha_{i}=\left\langle\phi^{-1}\left(\phi\left(\mu_{\alpha_{p}}\right)+\sum_{\substack{i=1 \\ i \neq p}}^{n} \phi\left(\mu_{\alpha_{i}}\right)\right), 1-\phi^{-1}\left(\phi\left(1-\nu_{\alpha_{p}}\right)+\sum_{\substack{i=1 \\ i \neq p}}^{n} \phi\left(1-\nu_{\alpha_{i}}\right)\right)\right\rangle$

Let $\alpha_{p}=\left\langle\mu_{\alpha_{p}}, \nu_{\alpha_{p}}\right\rangle=\langle 0,1\rangle$ i.e., $\mu_{\alpha_{p}}=0$ and $\nu_{\alpha_{p}}=1$. Then, using the operational law (7),

$\otimes_{c^{n}=1}^{n} \alpha_{i}=\left\langle\phi^{-1}\left(\phi(0)+\sum_{\substack{i=1 \\ i \neq p}}^{n} \phi\left(\mu_{\alpha_{i}}\right)\right), 1-\phi^{-1}\left(\begin{array}{c}\phi(1-1)+\sum_{\substack{i=1 \\ i \neq p}}^{n} \phi\left(1-\nu_{\alpha_{i}}\right)\end{array}\right)\right\rangle$

$=\left\langle\phi^{-1}\left(\phi(0)+\sum_{\substack{i=1 \\ i \neq p}}^{n} \phi\left(\mu_{\alpha_{i}}\right)\right), 1-\phi^{-1}\left(\phi(0)+\sum_{\substack{i=1 \\ i \neq p}}^{n} \phi\left(1-\nu_{\alpha_{i}}\right)\right)\right\rangle$

Using the existing relation $\phi(0)=\infty[1$, Proof of Theorem 2, p. 613],

$\otimes_{c_{i=1}^{n}}^{n} \alpha_{i}=\left\langle\phi^{-1}\left(\infty+\sum_{\substack{i=1 \\ i \neq p}}^{n} \phi\left(\mu_{\alpha_{i}}\right)\right), 1-\phi^{-1}\left(\begin{array}{c}\infty+\sum_{\substack{i=1 \\ i \neq p}}^{n} \phi\left(1-\nu_{\alpha_{i}}\right)\end{array}\right)\right\rangle$ $=\left\langle\phi^{-1}(\infty), 1-\phi^{-1}(\infty)\right\rangle$

Using the existing relation $\phi^{-1}(\infty)=0[1$, Proof of Theorem 2, p. 613],

$\otimes_{c i=1}^{n} \alpha_{i}=\langle 0,1-0\rangle$

$=\langle 0,1\rangle$.

\section{Limitation of Tao et al.'s IFCAAO}

In this section, it is shown that if one of the $n$ IFVs $\alpha_{i}=\left\langle\mu_{\alpha_{i}}, \nu_{\alpha_{i}}\right\rangle, i=1,2, \ldots, n$ will be $\langle 1,0\rangle$, then the aggregated IFV, obtained by Tao et al.'s IFCAAO, will also be $\langle 1$, $0\rangle$, i.e., if $\alpha_{p}=\langle 1,0\rangle$, then the aggregated IFV will be independent from the remaining $(n-1)$ IFV values $\alpha_{i}=\left\langle\mu_{\alpha_{i}}, \nu_{\alpha_{i}}\right\rangle$, $i=1,2, \ldots, p-1, p+1, \ldots, n$. Hence, the IFCAAO (5), proposed by Tao et al. [1] to aggregate $\alpha_{i}=\left\langle\mu_{\alpha_{i}}, \nu_{\alpha_{i}}\right\rangle, i=1,2$, $\ldots, n$, can be used only if $\alpha_{i} \neq\langle 1,0\rangle$ for any $i$.

The IFCAAO (8) represents an alternative form of the IFCAAO (5).

$$
\begin{aligned}
& \oplus_{c_{i=1}^{n}}^{n}\left(w_{i} \otimes_{c} \alpha_{i}\right)=\left\langle 1-\phi^{-1}\left(w_{p} \times \phi\left(1-\mu_{\alpha_{p}}\right)+\sum_{\substack{i=1 \\
i \neq p}}^{n}\left(w_{i} \times \phi\left(1-\mu_{\alpha_{i}}\right)\right)\right), \phi^{-1}\right. \\
& \left.\left(w_{p} \times \phi\left(\nu_{\alpha_{p}}\right)+\sum_{\substack{i=1 \\
i \neq p}}^{n}\left(w_{i} \times \phi\left(\nu_{\alpha_{i}}\right)\right)\right)\right)
\end{aligned}
$$

Let $\alpha_{p}=\left\langle\mu_{\alpha_{p}}, \nu_{\alpha_{p}}\right\rangle=\langle 1,0\rangle$, i.e., $\mu_{\alpha_{p}}=1$ and $\nu_{\alpha_{p}}=0$. Then, using the IFCAAO (8), 


$$
\begin{aligned}
\oplus_{c i=1}^{n}\left(w_{i} \otimes_{c} \alpha_{i}\right)= & \left\langle 1-\phi^{-1}\left(w_{p} \times \phi(1-1)+\sum_{\substack{i=1 \\
i \neq p}}^{n} w_{i} \phi\left(1-\mu_{\alpha_{i}}\right)\right), \phi^{-1}\right. \\
& \left.\left(\begin{array}{c}
w_{p} \times \phi(0)+\sum_{\substack{i=1 \\
i \neq p}}^{n} w_{i} \times \phi\left(\nu_{\alpha_{i}}\right)
\end{array}\right)\right\rangle
\end{aligned}
$$$$
=\left\langle 1-\phi^{-1}\left(w_{p} \times \phi(0)+\sum_{\substack{i=1 \\ i \neq p}}^{n} w_{i} \phi\left(1-\mu_{\alpha_{i}}\right)\right), \phi^{-1}\right.
$$$$
\left.\left(w_{p} \times \phi(0)+\sum_{\substack{i=1 \\ i \neq p}}^{n} w_{i} \times \phi\left(\nu_{\alpha_{i}}\right)\right)\right\rangle
$$

Using the existing relation $\phi(0)=\infty[1$, Proof of Theorem 2, p. 613],

$$
\begin{aligned}
& \oplus_{c i=1}^{n}\left(w_{i} \otimes_{c} \alpha_{i}\right)=\left\langle 1-\phi^{-1}\left(\begin{array}{c}
w_{p} \times \infty+\sum_{i=1}^{n}\left(w_{i} \times \phi\left(1-\mu_{\alpha_{i}}\right)\right) \\
i \neq p
\end{array}\right), \phi^{-1}\right. \\
& \left.\left(\begin{array}{c}
w_{p} \times \infty+\sum_{\substack{i=1 \\
i \neq p}}^{n}\left(w_{i} \times \phi\left(\nu_{\alpha_{i}}\right)\right)
\end{array}\right)\right\rangle \\
& =\left\langle 1-\phi^{-1}\left(\begin{array}{c}
\infty+\sum_{i=1}^{n}\left(w_{i} \times \phi\left(1-\mu_{\alpha_{i}}\right)\right) \\
i \neq p
\end{array}\right), \phi^{-1}\right. \\
& \left.\left(\begin{array}{c}
\infty+\sum_{i=1}^{n}\left(w_{i} \times \phi\left(\nu_{\alpha_{i}}\right)\right) \\
i \neq p
\end{array}\right)\right\rangle \\
& =\left\langle 1-\phi^{-1}(\infty), \phi^{-1}(\infty)\right\rangle
\end{aligned}
$$

Using the existing relation $\phi^{-1}(\infty)=0[1$, Proof of Theorem 2, p. 613],

$\oplus_{c i=1}^{n}\left(w_{i} \otimes_{c} \alpha_{i}\right)=\langle 1-0,0\rangle$

$=\langle 1,0\rangle$.

\section{Impact of the Limitation of Tao et al.'s IFCAAO on the Ranking of the Alternatives of an Existing Multiple-Attribute Decision-Making Problem}

Tao et al. [1] considered a multiple-attribute decision-making problem of the ranking of four projectors to illustrate their proposed IFCAAO.

In this section, firstly, the multiple-attribute decision-making problem, considered by the Tao et al. [1], is discussed in a brief manner. Then, the impact of the limitation of Tao et al.'s IFCAAO [1] on the ranking of alternatives of multipleattribute decision-making problem, considered by Tao et al. [1], is discussed.

\section{A Brief Review of Tao et al.'s Multiple-Attribute Decision-Making Problem}

Tao et al. [1] used the following method to rank the four projectors by considering that the $(i, j)$ th IFV of the intuitionistic fuzzy decision matrix $\widetilde{D}=\left(\alpha_{i j}\right)_{4 \times 5}=\left(\begin{array}{ccccc}\langle 0.4,0.3\rangle & \langle 0.5,0.2\rangle & \langle 0.7,0.2\rangle & \langle 0.4,0.6\rangle & \langle 0.6,0.2\rangle \\ \langle 0.6,0.1\rangle & \langle 0.4,0.3\rangle & \langle 0.3,0.5\rangle & \langle 0.6,0.2\rangle & \langle 0.5,0.3\rangle \\ \langle 0.5,0.4\rangle & \langle 0.6,0.1\rangle & \langle 0.6,0.2\rangle & \langle 0.7,0.1\rangle & \langle 0.3,0.6\rangle \\ \langle 0.6,0.3\rangle & \langle 0.4,0.5\rangle & \langle 0.5,0.3\rangle & \langle 0.8,0.2\rangle & \langle 0.5,0.2\rangle\end{array}\right)$ represents the rating value of the $i$ th projector $(i=1,2,3,4)$ over the $j$ th benefit attribute $(j=1,2,3,4,5)$, provided by a decision-maker.

Step 1: Tao et al. [1] applied an existing method to evaluate the normalized weights $w_{1}, w_{2}, w_{3}, w_{4}$, and $w_{5}$ corresponding to the first, second, third, fourth, and fifth attributes respectively.

Step 2: Tao et al. [1] applied the IFCAAO to evaluate

(i) The IFV $\alpha_{1}=w_{1} \otimes_{c}\langle 0.4,0.3\rangle \oplus_{c} w_{2} \otimes_{c}\langle 0.5,0.2\rangle \oplus_{c} w_{3} \otimes-$ ${ }_{c}\langle 0.7,0.2\rangle \oplus_{c} w_{4} \otimes_{c}\langle 0.4,0.6\rangle \oplus_{c} w_{5} \otimes_{c}\langle 0.6,0.2\rangle$ corresponding to the first projector.

(ii) The IFV $\alpha_{2}=w_{1} \otimes_{c}\langle 0.6,0.1\rangle \oplus_{c} w_{2} \otimes_{c}\langle 0.4,0.3\rangle \oplus_{c} w_{3} \otimes-$ ${ }_{c}\langle 0.3,0.5\rangle \oplus_{c} w_{4} \otimes_{c}\langle 0.6,0.2\rangle \oplus_{c} w_{5} \otimes_{c}\langle 0.5,0.3\rangle$ corresponding to the second projector.

(iii) The IFV $\alpha_{3}=w_{1} \otimes_{c}\langle 0.5,0.4\rangle \oplus_{c} w_{2} \otimes_{c}\langle 0.6,0.1\rangle \oplus_{c} w_{3} \otimes-$ ${ }_{c}\langle 0.6,0.2\rangle \oplus_{c} w_{4} \otimes_{c}\langle 0.7,0.1\rangle \oplus_{c} w_{5} \otimes_{c}\langle 0.3,0.6\rangle$ corresponding to the third projector.

(iv) The IFV $\alpha_{4}=w_{1} \otimes_{c}\langle 0.6,0.3\rangle \oplus_{c} w_{2} \otimes_{c}\langle 0.4,0.5\rangle \oplus_{c} w_{3} \otimes-$ ${ }_{c}\langle 0.5,0.3\rangle \oplus_{c} w_{4} \otimes_{c}\langle 0.8,0.2\rangle \oplus_{c} w_{5} \otimes_{c}\langle 0.5,0.2\rangle$ corresponding to the fourth projector.

Step 3: Tao et al. [1] used the following method to conclude that that the $p$ th alternative is better than the $q$ th alternative or vice versa.

Step (3a): Check Score $\left(\alpha_{p}\right)>$ Score $\left(\alpha_{q}\right)$ or Score $\left(\alpha_{p}\right)<$ Score $\left(\alpha_{q}\right)$ or Score $\left(\alpha_{p}\right)=\operatorname{Score}\left(\alpha_{q}\right)$,

where,

Score $\left(\alpha_{p}\right)=\operatorname{Score}\left(\left\langle\mu_{\alpha_{p}}, \nu_{\alpha_{p}}\right\rangle\right)=\mu_{\alpha_{p}}-\nu_{\alpha_{p}} \quad$ a n d Score $\left(\alpha_{q}\right)=\operatorname{Score}\left(\left\langle\mu_{\alpha_{q}}, \nu_{\alpha_{q}}\right\rangle\right)=\mu_{\alpha_{q}}-\nu_{\alpha_{q}}$.

Case (i): If Score $\left(\alpha_{p}\right)>$ Score $\left(\alpha_{q}\right)$, then the $p$ th alternative is better than the $q$ th alternative.

Case (ii): If Score $\left(\alpha_{p}\right)<$ Score $\left(\alpha_{q}\right)$, then the $q$ th alternative is better than the $p$ th alternative.

Case (iii): If Score $\left(\alpha_{p}\right)=$ Score $\left(\alpha_{q}\right)$, then go to step (3b).

Step (3b): Check Accuracy $\left(\alpha_{p}\right)>$ Accuracy $\left(\alpha_{q}\right)$ or Accuracy $\left(\alpha_{p}\right)<\operatorname{Accuracy}\left(\alpha_{q}\right)$, where, 
$\operatorname{Accuracy}\left(\alpha_{p}\right)=\operatorname{Accuracy}\left(\left\langle\mu_{\alpha_{p}}, \nu_{\alpha_{p}}\right\rangle\right)=\mu_{\alpha_{p}}+\nu_{\alpha_{p}}$ and

$\operatorname{Accuracy}\left(\alpha_{q}\right)=\operatorname{Accuracy}\left(\left\langle\mu_{\alpha_{q}}, \nu_{\alpha_{q}}\right\rangle\right)=\mu_{\alpha_{q}}+\nu_{\alpha_{q}}$.

Case (i): If Accuracy $\left(\alpha_{p}\right)>$ Accuracy $\left(\alpha_{q}\right)$, then the $p$ th alternative is better than the $q$ th alternative.

Case (ii): If Accuracy $\left(\alpha_{p}\right)<$ Accuracy $\left(\alpha_{q}\right)$, then the $q$ th alternative is better than the $p$ th alternative.

Case (iii): If Accuracy $\left(\alpha_{p}\right)=$ Accuracy $\left(\alpha_{q}\right)$, then the $p$ th alternative is equivalent to the $q$ th alternative.

\section{Impact of the Limitation of Tao et al.'s IFCAAO}

It is obvious from "A Brief Review of Tao Et al.'s MultipleAttribute Decision-Making Problem" that in step 2, the IFCAAO (5) has been used to obtain

(i) The IFV $\alpha_{1}=w_{1} \otimes_{c}\langle 0.4,0.3\rangle \oplus_{c} w_{2} \otimes_{c}\langle 0.5,0.2\rangle \oplus_{c} w_{3} \otimes-$ ${ }_{c}\langle 0.7,0.2\rangle$

$\oplus_{c} w_{4} \otimes_{c}\langle 0.4,0.6\rangle \oplus_{c} w_{5} \otimes_{c}\langle 0.6,0.2\rangle$ corresponding to the first projector.

If the IFV $\alpha_{11}=\langle 0.4,0.3\rangle$ or the IFV $\alpha_{12}=\langle 0.5,0.2\rangle$ or the IFV $\alpha_{13}=\langle 0.7,0.2\rangle$ or the IFV $\alpha_{14}=\langle 0.4,0.6\rangle$ or the IFV $\alpha_{15}=\langle 0.6,0.2\rangle$ is replaced by the IFV $\langle 1,0\rangle$. Then, according to "Limitation of Tao et al.'s IFCAAO," the obtained IFV $\alpha_{1}$ will be $\langle 1,0\rangle$.

Furthermore, as $\langle 1,0\rangle$ is the only IFV for which Score will be 1 and the Score of any IFV can never be more than 1 , in such a situation, the first projector will be one of the best projectors, i.e., if $\alpha_{1 j}=\langle 1,0\rangle$ for any $j$, then the result "the first projector is one of the best projectors" is independent from all the remaining 19 IFVs of the intuitionistic fuzzy decision matrix $\widetilde{D}$.

(ii) The IFV $\alpha_{2}=w_{1} \otimes_{c}\langle 0.6,0.1\rangle \oplus_{c} w_{2} \otimes_{c}\langle 0.4,0.3\rangle \oplus_{c} w_{3} \otimes-$ ${ }_{c}\langle 0.3,0.5\rangle \oplus_{c}$

$w_{4} \otimes_{c}\langle 0.6,0.2\rangle \oplus_{c} w_{5} \otimes_{c}\langle 0.5,0.3\rangle$, corresponding to the second projector.

If the IFV $\alpha_{21}=\langle 0.6,0.1\rangle$ or the IFV $\alpha_{22}=\langle 0.4,0.3\rangle$ or the IFV $\alpha_{23}=\langle 0.3,0.5\rangle$ or the IFV $\alpha_{24}=\langle 0.6,0.2\rangle$ or the IFV $\alpha_{25}=\langle 0.5,0.3\rangle$ is replaced by the IFV $\langle 1,0\rangle$, then, according to "Limitation of Tao et al.'s IFCAAO," the obtained IFV $\alpha_{2}$ will be $\langle 1,0\rangle$.

Furthermore, as discussed in (i), in such a situation, the second projector will be one of the best projectors, i.e., if $\alpha_{2 j}=\langle 1,0\rangle$ for any $j$, then the result "the second projector is one of the best projectors" is independent from all the remaining 19 IFVs of the intuitionistic fuzzy decision matrix $\widetilde{D}$.

(iii) The IFV $\alpha_{3}=w_{1} \otimes_{c}\langle 0.5,0.4\rangle \oplus_{c} w_{2} \otimes_{c}\langle 0.6,0.1\rangle \oplus_{c} w_{3} \otimes-$ ${ }_{c}\langle 0.6,0.2\rangle \oplus_{c} w_{4} \otimes_{c}\langle 0.7,0.1\rangle \oplus_{c} w_{5} \otimes_{c}\langle 0.3,0.6\rangle$ corresponding to the third projector.

If the IFV $\alpha_{31}=\langle 0.5,0.4\rangle$ or the IFV $\alpha_{32}=\langle 0.6,0.1\rangle$ or the IFV $\alpha_{33}=\langle 0.6,0.2\rangle$ or the IFV $\alpha_{34}=\langle 0.7,0.1\rangle$ or the IFV $\alpha_{35}=\langle 0.3,0.6\rangle$ is replaced by the IFV $\langle 1,0\rangle$, then, according to "Limitation of Tao et al.'s IFCAAO," the obtained IFV $\alpha_{3}$ will be $\langle 1,0\rangle$.

Furthermore, as discussed in (i), in such a situation, the third projector will be one of the best projectors, i.e., if $\alpha_{3 j}=\langle 1,0\rangle$ for any $j$, then the result "the third projector is one of the best projectors" is independent from all the remaining 19 IFVs of the intuitionistic fuzzy decision matrix $\widetilde{D}$.

(iv) The IFV $\alpha_{4}=w_{1} \otimes_{c}\langle 0.6,0.3\rangle \oplus_{c} w_{2} \otimes_{c}\langle 0.4,0.5\rangle \oplus_{c} w_{3} \otimes-$ ${ }_{c}\langle 0.5,0.3\rangle$

$\oplus_{c} w_{4} \otimes_{c}\langle 0.8,0.2\rangle \oplus_{c} w_{5} \otimes_{c}\langle 0.5,0.2\rangle$, corresponding to the fourth projector.

If the IFV $\alpha_{41}=\langle 0.6,0.3\rangle$ or the IFV $\alpha_{42}=\langle 0.4,0.5\rangle$ or the IFV $\alpha_{43}=\langle 0.5,0.3\rangle$ or the IFV $\alpha_{44}=\langle 0.8,0.2\rangle$ or the IFV $\alpha_{45}=\langle 0.5,0.2\rangle$ is replaced by the IFV $\langle 1,0\rangle$, then, according to "Limitation of Tao et al.'s IFCAAO," the obtained IFV $\alpha_{4}$ will be $\langle 1,0\rangle$.

Furthermore, as discussed in (i), in such a situation, the fourth projector will be one of the best projectors, i.e., if $\alpha_{4 j}=\langle 1,0\rangle$ for any $j$, then the result "the fourth projector is one of the best projectors" is independent from all the remaining 19 IFVs of the intuitionistic fuzzy decision matrix $\widetilde{D}$.

\section{Conclusion}

It is shown that the operational law (3) to evaluate sum of finite number of IFVs and the IFCAAO (5) to aggregate finite number of IFVs, proposed by Tao et al. [1], can be used only if $\alpha_{i} \neq\langle 1,0\rangle$ for any $i$. Also, it is shown that the operational law (4) to evaluate the multiplication of finite number of IFVs, proposed by Tao et al. [1], can be used only if $\alpha_{i} \neq\langle 0,1\rangle$ for any $i$.

Acknowledgments The authors would like to thank the Editor-in-Chief "Professor Amir Hussain" and the anonymous reviewers for their constructive suggestions which have led to an improvement in both the quality and the clarity of the paper. 


\section{Compliance with Ethical Standards}

Conflict of Interest The authors declare that they have no conflict of interest.

Ethical Approval This article does not contain any studies with human participants or animals performed by any of the authors.

\section{Reference}

1. Tao Z, Bing H, Chen H. On intuitionistic fuzzy copula aggregation operators in multiple-attribute decision making. Cong Comput. 2018;10:610-24.

Publisher's Note Springer Nature remains neutral with regard to jurisdictional claims in published maps and institutional affiliations. 